
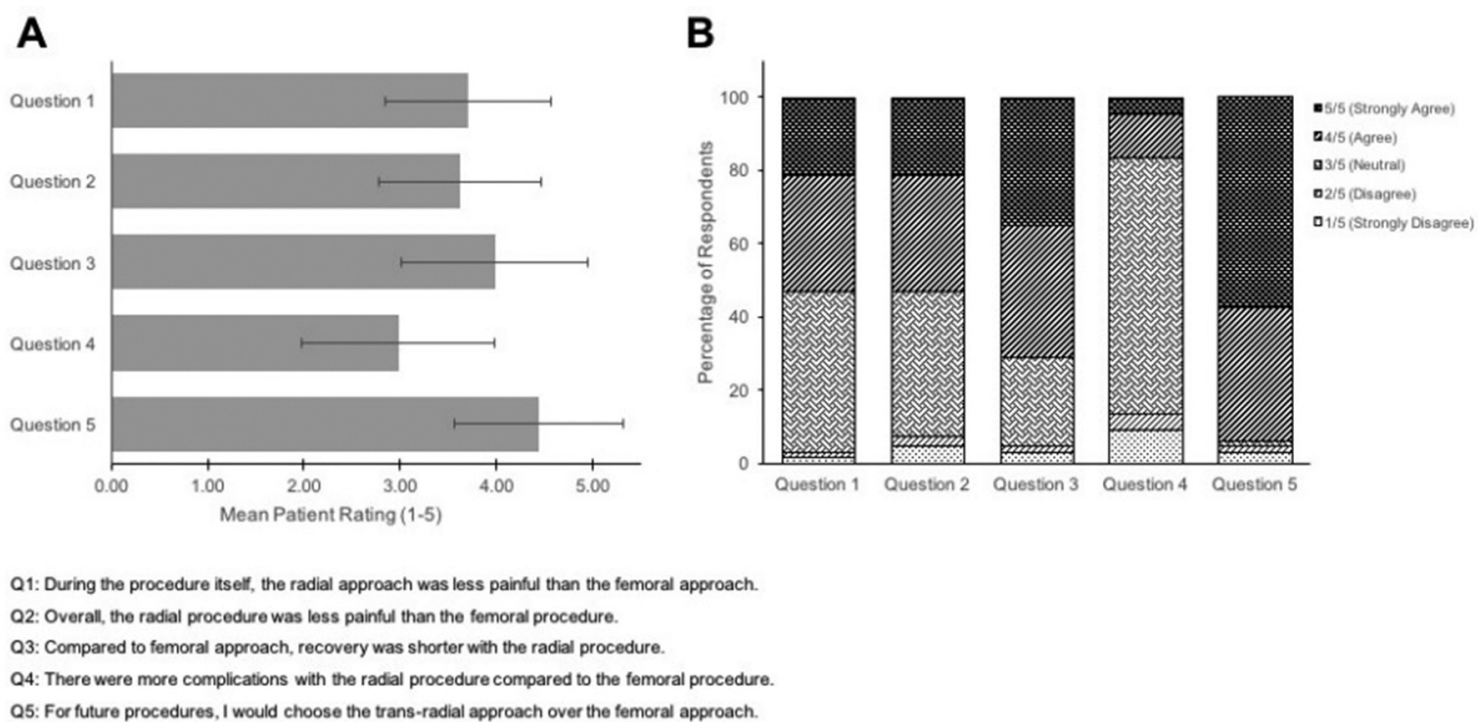

Abstract E-053 Figure 1

\author{
Abstract E-053 Table 1 \\ Total patients: 223 \\ Male: 104/Female: 119 \\ Mean age: 61 years old (range 23-96) \\ Total procedures performed via radial artery catheterization: 233 \\ Cerebral angiogram (diagnostic): 172 \\ Aneurysm stent/coil: 33 \\ Carotid-cavernous fistula embolization: 2 \\ Dural arterio-venous fistula embolization: 2 \\ Mechanical thrombectomy: 1412 anterior circulation/2 posterior circulation \\ Carotid stent placement: 7 \\ Radiosurgery: 3 \\ Vascular access complications: 10 \\ Unable to obtain radial artery catheterization: 6 \\ Radial artery spasm: 2 \\ Switch to groin access to complete procedure: 10 \\ latrogenic complications (dissection, hemorrhage, stroke): none \\ Post-op complications: 10 \\ Superficial hematoma: 5 \\ Ecchymosis: 2 \\ Swelling/pain/paresthesias: 3 \\ Post-op complications seen at follow-up visit: 5 \\ Superficial hematoma: 2 \\ Ecchymosis: 2 \\ Swelling/pain/paresthesias: 1 \\ Superficial thrombophlebitis: 1
}

incidence of perioperative and post-procedural complications was investigated. We identified a subset of 98 patients who have undergone both transradial and transfemoral cerebral angiograms, and compared clinical outcomes and patient satisfaction measures between the two groups.

Results The overall incidence of complications was low across all procedures performed via transradial access. Peri-procedurally, only 2 patients had symptomatic radial artery spasm, and there were no instances of iatrogenic complications (vessel dissection, stroke, hemorrhage). In 10 cases $(4.3 \%)$, the intended procedure could not be completed via a transradial approach, and, thus, femoral artery access had to be pursued instead.
Ten patients complained of minor post-procedural complications: superficial hematomas, swelling, and paresthesias, albeit none required therapeutic intervention. The mean procedure time was shorter for diagnostic angiograms performed via transradial vs transfemoral access $(18.8 \pm 15.8$ versus 39.5 \pm 31.1 minutes, $p<0.05)$. Patients reported shorter recovery times with transradial access, and the majority of patients (94\%) would elect to have subsequent procedures performed via this route.

Conclusions Radial artery catheterization is a safe and durable alternative to perform a wide range of neuroendovascular procedures, with a low rate of complications. On the whole, patients prefer transradial compared to transfemoral access, with shorter recovery times reported and overall excellent patient satisfaction.

Disclosures O. Khanna: None.

\section{E-054 INTERPRETABILITY OF AUTOMATED COMPUTED TOMOGRAPHY PERFUSION FOR STROKE THROMBECTOMY}

${ }^{1} \mathrm{Z}$ Bulwa*, ${ }^{2} \mathrm{H}$ Dasenbrock, ${ }^{3} \mathrm{~N}$ Osteraas, ${ }^{3} \mathrm{~L}$ Cherian, ${ }^{2} \mathrm{R}$ Crowley, ${ }^{2} \mathrm{M}$ Chen. ${ }^{1}$ Neurology, University of Chicago, Chicago, IL; ${ }^{2}$ Neurosurgery, Rush University, Chicago, IL; ${ }^{3}$ Neurology, Rush University, Chicago, IL

\subsection{6/neurintsurg-2019-SNIS.129}

Introduction Landmark trials have demonstrated that select patients with anterior large vessel occlusions (LVO) may benefit from thrombectomy past previously established timeframes. The automated computed tomography perfusion (CTP) software used in these trials is costly, not widely available, and, at times, difficult to interpret. Despite the increasing adoption of automated CTP, feasibility issues exist. Our study aims to critically assess an automated CTP program in a large, contemporary cohort of patients presenting with LVO in the extended treatment window by evaluating the rate of uninterpretable studies and influence on clinical outcomes.

Methods A two-year retrospective review of consecutive thrombectomy candidates in the extended time window at an urban, academic, tertiary care comprehensive stroke center 
was performed. CTP imaging was employed to select appropriate patients for thrombectomy who had presented greater than six hours after symptom onset or with unknown time of symptom onset. Automated CTP maps using RAPID (iSchemaView, Menlo Park, CA) software were technically adequate if the hypoperfused ischemic penumbra and infarcted core could be characterized visually by the acute stroke team and used to inform patient selection. Clinical outcomes were compared between patients who had uninterpretable automated CTP maps and those with interpretable studies.

Results 99 consecutive patients underwent automated CTP imaging from February 2017 through December 2018 for possible thrombectomy. Of these, 78 (79\%) had LVO determined by CT angiography (CTA) and were included in the study cohort. Automated CTP maps were uninterpretable in $13 \%$ of cases $(n=10)$. Reasons for uninterpretable automated CTP maps included motion artifact $(n=3)$ and contrast bolus failure $(\mathrm{n}=7)$. There were trends approaching significance of younger age (57 years (IQR 47-57) vs 68 years (IQR 55-79); $p=0.12$ ) and a history of heart failure with reduced ejection fraction (30\% vs $9 \% ; p=0.09)$ in the uninterpretable group versus the interpretable group, (Table1). Eight patients with uninterpretable automated CTP maps had ASPECTS $\geq 7$; all of these patients underwent thrombectomy. The remaining two patients with uninterpretable imaging had ASPECTS $\leq 5$ and did not undergo thrombectomy. In patients undergoing thrombectomy after automated CTP imaging, median NIHSS on presentation (18 (16-21) vs $19(16-23) ; p=1.0)$, ASPECTS (8 (7-9) vs 8 $(6-9) ; p=0.65)$ and discharge to home/acute rehab $(75 \%$ vs. $89 \% ; p=0.58)$ did not significantly differ with regard to CTP interpretability. [Table 2]. Additionally, a substantial population of patients with interpretable CTP studies had discrepancies between ASPECTS and automated CTP infarct core. Twenty five patients with interpretable automated CTP maps had an estimated infarct core of $0 \mathrm{~mL}$ and $44 \%$ of these patients had ASPECTS $<10(n=11)$.

Conclusion CTP maps generated by RAPID software were uninterpretable in $13 \%$ of cases within our cohort; more commonly in patients with reduced ejection fraction and younger age. This is a higher rate than prior publications. Given the substantial rate of uninterpretable automated CTP maps, further studies are warranted to evaluate more reliable and readily available methods to select patients for thrombectomy who present in the extended time window.

Disclosures Z. Bulwa: None. H. Dasenbrock: None. N. Osteraas: None. L. Cherian: None. R. Crowley: None. M. Chen: None.

\section{E-055 FLAIR HYPERINTENSITIES ON MRI IN M2 OCCLUSION}

${ }^{1} \mathrm{~K}$ Dakay*, ${ }^{2} \mathrm{M}$ Jayaraman, ${ }^{2} \mathrm{R}$ McTaggart, ${ }^{1} \mathrm{~S}$ Yaghi, ${ }^{3} \mathrm{G}$ Jindal, ${ }^{1} \mathrm{~S}$ Cutting. ${ }^{1}$ Neurology, Warren Alpert Medical School of Brown University, Providence, Rl; ${ }^{2}$ Diagnostic Imaging, Neurosurgery, Warren Alpert Medical School of Brown University, Providence, Rl: ${ }^{3}$ Diagnostic Imaging, Warren Alpert Medical School of Brown University, Providence, $R I$

\subsection{6/neurintsurg-2019-SNIS.130}

Background M2 segment occlusion of the middle cerebral artery is a potentially debilitating cause of stroke, and the data is unclear on the optimal method of treatment for these patients. FLAIR hyperintense vessels (FHV) on MRI have been postulated to be correlated with ischemic penumbra, and we sought to evaluate the incidence of this radiologic finding in patients with M2 occlusion. Additionally, the prognosis of

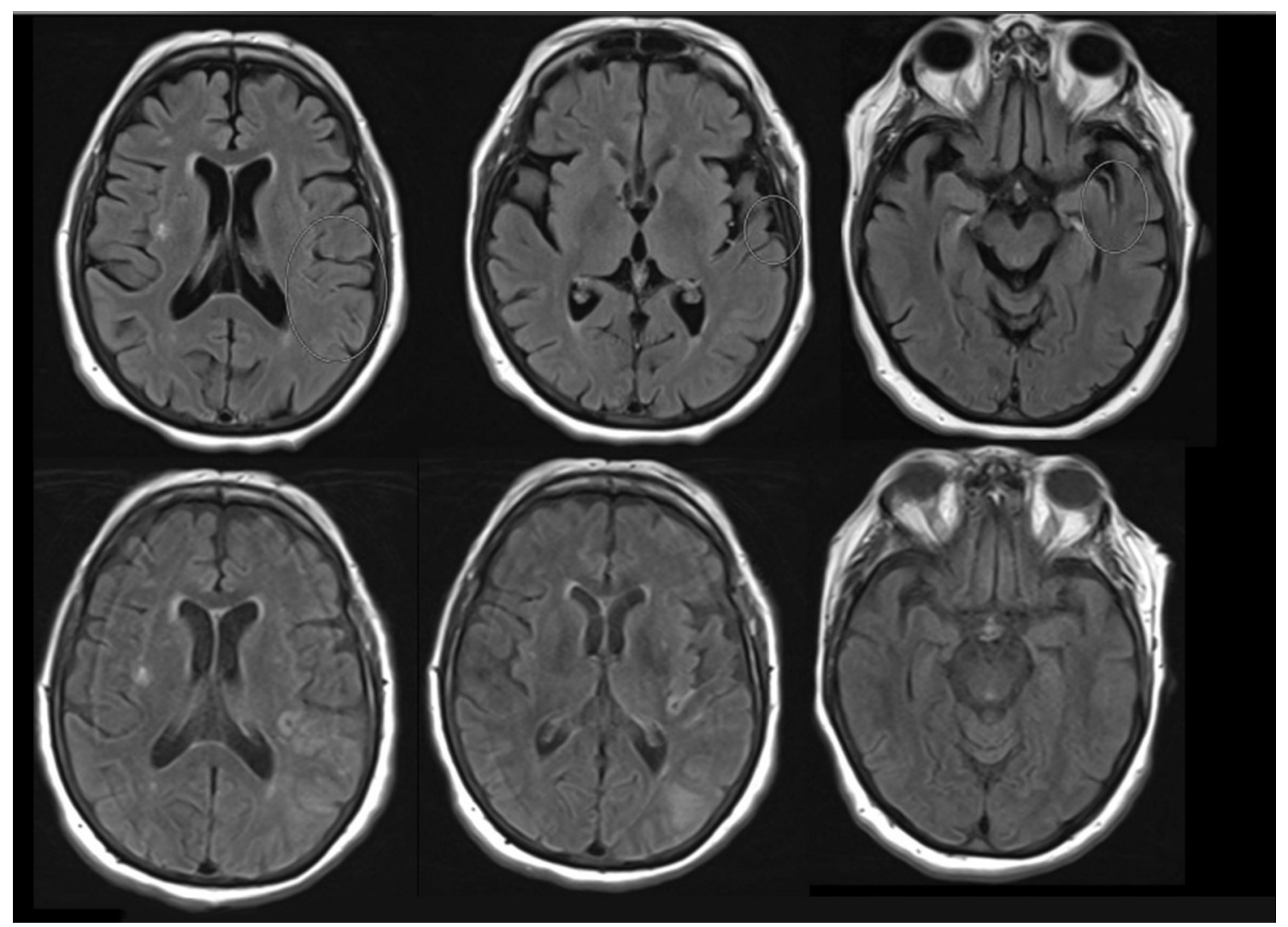

Abstract E-055 Figure 1 Pre-treatment MRI showed extensive FLAIR hyperintense vessels. Repeat imaging one day after endovascular therapy was artifact-limited, but showed resolution of the FLAIR hyperintense vessels. 